\title{
Machine learning analysis to identify the association between risk factors and onset of nosocomial diarrhea: a retrospective cohort study
}

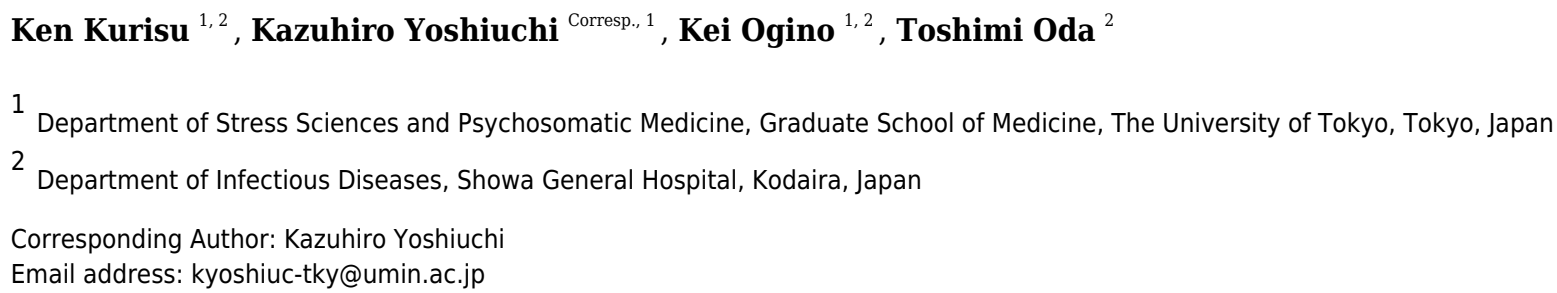

Background. Although several risk factors for nosocomial diarrhea have been identified, the detail of association between these factors and onset of nosocomial diarrhea, such as degree of importance or temporal pattern of influence, remains unclear. We aimed to determine the association between risk factors and onset of nosocomial diarrhea using machine learning algorithms. Methods. We retrospectively collected data of patients with acute cerebral infarction. Seven variables, including age, sex, modified Rankin Scale (mRS) score, and number of days of antibiotics, tube feeding, proton pump inhibitors, and histamine 2-receptor antagonist use, were used in the analysis. We split the data into a training dataset and independent test dataset. Based on the training dataset, we developed a random forest, support vector machine (SVM), and radial basis function (RBF) network model. By calculating an area under the curve (AUC) of the receiver operating characteristic curve using 5-fold cross-validation, we performed feature selection and hyperparameter optimization in each model. According to their final performances, we selected the optimal model and also validated it in the independent test dataset. Based on the selected model, we visualized the variable importance and the association between each variable and the outcome using partial dependence plots. Results. Two-hundred and eighteen patients were included. In the cross-validation within the training dataset, the random forest model achieved an AUC of 0.944 , which was higher than in the SVM and RBF network models. The random forest model also achieved an AUC of 0.832 in the independent test dataset. Tube feeding use days, mRS score, antibiotic use days, age and sex were strongly associated with the onset of nosocomial diarrhea, in this order. Tube feeding use had an inverse U-shaped association with the outcome. The mRS score and age had a convex downward and increasing association, while antibiotic use had a convex upward association with the outcome. Conclusion. We revealed the degree of importance and temporal pattern of the influence of several risk factors for nosocomial diarrhea, which 
could help clinicians manage nosocomial diarrhea. 
1 Machine learning analysis to identify the association

2 between risk factors and onset of nosocomial

3 diarrhea: a retrospective cohort study

4

5 Ken Kurisu ${ }^{1,2}$, Kazuhiro Yoshiuchi ${ }^{2}$, Kei Ogino $^{1,2}$, Toshimi Oda ${ }^{1}$

$7 \quad{ }^{1}$ Department of Infectious Diseases, Showa General Hospital, 8-1-1 Hanakoganei, Kodaira,

8 Tokyo 187-8510, Japan.

$9 \quad{ }^{2}$ Department of Stress Sciences and Psychosomatic Medicine, Graduate School of Medicine,

10 The University of Tokyo, 7-3-1 Hongo, Bunkyo-ku, Tokyo 113-8655, Japan

11

12 Corresponding Author:

13 Kazuhiro Yoshiuchi ${ }^{2}, \mathrm{MD}, \mathrm{PhD}$

14 Department of Stress Sciences and Psychosomatic Medicine, Graduate School of Medicine, The

15 University of Tokyo, 7-3-1, Hongo, Bunkyo-ku, Tokyo 113-8655, Japan.

16 Email address: kyoshiuc-tky@umin.ac.jp 


\section{Abstract}

19 Background. Although several risk factors for nosocomial diarrhea have been identified, the 20 detail of association between these factors and onset of nosocomial diarrhea, such as degree of importance or temporal pattern of influence, remains unclear. We aimed to determine the association between risk factors and onset of nosocomial diarrhea using machine learning 23 algorithms.

24 Methods. We retrospectively collected data of patients with acute cerebral infarction. Seven 25 variables, including age, sex, modified Rankin Scale (mRS) score, and number of days of antibiotics, tube feeding, proton pump inhibitors, and histamine 2-receptor antagonist use, were used in the analysis. We split the data into a training dataset and independent test dataset. Based on the training dataset, we developed a random forest, support vector machine (SVM), and radial basis function (RBF) network model. By calculating an area under the curve (AUC) of the receiver operating characteristic curve using 5-fold cross-validation, we performed feature selection and hyperparameter optimization in each model. According to their final performances, we selected the optimal model and also validated it in the independent test dataset. Based on the selected model, we visualized the variable importance and the association between each variable and the outcome using partial dependence plots.

Results. Two-hundred and eighteen patients were included. In the cross-validation within the training dataset, the random forest model achieved an AUC of 0.944, which was higher than in the SVM and RBF network models. The random forest model also achieved an AUC of 0.832 in the independent test dataset. Tube feeding use days, mRS score, antibiotic use days, age and sex were strongly associated with the onset of nosocomial diarrhea, in this order. Tube feeding use had an inverse U-shaped association with the outcome. The mRS score and age had a convex downward and increasing association, while antibiotic use had a convex upward association with the outcome.

43 Conclusion. We revealed the degree of importance and temporal pattern of the influence of several risk factors for nosocomial diarrhea, which could help clinicians manage nosocomial 45 diarrhea. 


\section{Introduction}

48 Nosocomial diarrhea is a common problem among hospitalized patients. It increases length of

49 stay and healthcare costs (Kyne et al., 2002a) and is important for hospital infection control.

50 Several risk factors for nosocomial diarrhea have been identified, including history of

51 hospitalization, gastrointestinal surgery, severity of disease, and tube feeding, proton pump

52 inhibitor (PPI), histamine 2-receptor antagonist (H2RA), and antibiotic use (McFarland, 1995;

53 Kyne et al., 2002b; Thorson, Bliss \& Savik, 2008; Arevalo-Manso et al., 2014; Eze et al., 2017;

54 Thabit, Varugehese \& Levine, 2019).

55 However, details about association between these risk factors and the onset of nosocomial

56 diarrhea remains unclear. There are few reports about the association between the risk of onset of

57 nosocomial diarrhea and the duration of antibiotic, PPI, H2RA, and tube feeding use. Clinical

58

59

60

61

62

63

64

65

66

67

68

69

70

71

72

73

74

75

76

77

78

79

80

81

82

83

84

85

86

knowledge of relative importance or temporal pattern of the influence of risk factors for nosocomial diarrhea could result in minimizing administration of these drugs or considering administration of probiotics for preventing nosocomial diarrhea (Hempel et al., 2012, Goldenberg et al., 2017).

Recently, there have been many reports using machine learning algorithms in the medical field, such as random forest, support vector machine (SVM), or radial basis function (RBF) network (Bair et al., 2013; DuBrava et al., 2017; Kimura et al., 2019; Halladay, Sillner \& Rudolph, 2018; Le, Ho \& Ou, 2018; Le et al., 2019a; Tamune et al., 2019; Cho et al., 2018; Ding et al., 2018; Zarbakhsh \& Addeh, 2018). These models are non-linear and non-monotonous. They can deal with variables that have a complex association, such as a $U$-shaped or convex association, with the outcome. They can analyze the duration of exposure and presence or absence of exposure simultaneously. Traditional statistical models, such as a logistic regression or Cox regression model, do not have such properties. In addition, some machine learning models have high interpretability because they can visualize the variable importance (Breiman, 2001; Fisher, Rudin \& Dominici, 2018) or association between the variables and the outcome by partial dependence plots (Friedman, 2001).

We hypothesized that such machine learning algorithms could reveal the unknown association, such as degree of importance and temporal pattern of the influence of risk factors for nosocomial diarrhea, which would be helpful for clinicians. The present study aimed to determine the association between the risk factors and onset of nosocomial diarrhea using machine learning algorithms. We used the data of patients hospitalized with acute cerebral infarction because they often have several risk factors for nosocomial diarrhea, such as PPI, H2RA, and tube feeding use.

\section{Materials \& Methods}

\section{Ethics approval}

This study was approved by the Institutional Review Board of Showa General Hospital (approval number: REC-180). In the present study, because of the anonymous nature of the data and the non-invasive study, the requirement for informed consent was waived. Instead, we released the

Peer) reviewing PDF | (2019:06:38745:1:2:CHECK 31 Aug 2019) 
87 research project on the website of Showa General Hospital so that patients could reject utilization

88 of their data.

89

90

91

92

93

94

95

96

97

98

99

100

101

102

103

104

105

106

107

108

109

110

111

112

113

114

115

116

117

118

119

120

121

122

123

124

125

\section{Design and study population}

This retrospective cohort study was conducted in Showa General Hospital, a single tertiary center in Japan. We collected data from electronic medical records.

We included patients admitted from April 2017 to March 2018 for acute cerebral infarction, except for those diagnosed with subtype 4 infarction according to the Trial of Org 10172 in Acute Stroke Treatment (TOAST) classification (Adams et al., 1993), who stayed for more than 3 days. To our knowledge, there is no theoretical method to determine the sample size in machine learning models. Therefore, we included patients admitted during one fiscal year. Cerebral infarction of subtype 4 according to the TOAST classification includes diseases, such as vertebral artery dissection. Because cases of subtype 4 cerebral infarction are relatively rare (Kolominsky-Rabas et al., 2001), we did not use them to reduce patient heterogeneity. The present study focused on nosocomial diarrhea, which occurs more than 3 days after admission (Bauer et al., 2001). Therefore, patients who stayed for more than 3 days were eligible. The exclusion criteria were history of abdominal surgery, gastrointestinal disease as a comorbidity, regular use of laxatives, and the onset of diarrhea within 3 days from admission. Patients with a history of abdominal surgery and those with gastrointestinal disease as a comorbidity were excluded to streamline the analysis. Patients who were using laxatives regularly were excluded because it was difficult to conclude whether diarrhea in these patients was caused by the regular use of laxatives. Patients who presented with diarrhea within 3 days from admission were excluded for the same reason as those who left the hospital within 3 days from admission.

\section{Outcome}

According to the criteria established by the World Health Organization (2018) and used in previous research (Arevalo-Manso et al., 2014), diarrhea was defined as the passage of 3 or more liquid stools or stools of types 5 to 7 according to the Bristol Stool Form Scale (Lewis \& Heaton, 1997) within 24 hours. If patients had diarrhea but were using laxatives temporarily within 3 days of the onset of diarrhea, we considered that their diarrhea was likely to be caused by the laxatives, regardless of the risk factors, and we categorized them into the non-diarrhea group. The observational period was from admission to the onset of diarrhea in patients who had diarrhea and from admission to discharge in patients who did not have diarrhea.

We used C. Diff Quik Chek (Abbott, Lake Bluff, Ill.), a tool of enzyme immunoassay for Clostridioides difficile (CD) glutamate dehydrogenase (GDH) antigen and CD toxins A and B, as the diagnostic tool for CD infection (CDI). It is reported that sensitivity and specificity for GDH antigen is over $90 \%$, sensitivity for CD toxin is almost $50 \%$, and specificity for CD toxin is over 90\% in Japanese hospitals (Kawada et al., 2011; Kosai et al., 2017; Morinaga et al., 2018). 
127 Variables

128 The following 7 variables were considered as risk factors or confounding factors for nosocomial 129 diarrhea and were included in the analysis: age, sex, severity of illness, and number of days of 130 use of tube feeding, PPI, H2RA, and antibiotics (McFarland, 1995; Kyne et al., 2002b; Thorson, 131 Bliss \& Savik, 2008; Arevalo-Manso et al., 2014; Eze et al., 2017; Thabit, Varugehese \& Levine, 132 2019). The modified Rankin Scale (mRS) (van Swieten et al., 1988) was used as the index of 133 disease severity and was scored 2 days after admission. The number of days of exposure to the 134 risk factors was calculated at the end of the observational period: at the onset of diarrhea for 135 patients in the diarrhea group and at discharge for patients in the non-diarrhea group. For patients 136 without exposure, each variable was set to 0.

137

138

\section{Analysis of demographic data}

139

140

All analyses were conducted using the open source software R version 3.6.1 (R Foundation for

141 Statistical Computing, Vienna, Austria). The threshold for statistical significance was set to $P<$ .05 .

We used the Student's $t$ test to compare the averages of continuous variables and the chi-squared test to compare the proportions of categorical variables between the diarrhea and non-diarrhea groups. The multicollinearity of the independent variables was evaluated using variance inflation factors (VIFs).

\section{Model selection}

We developed machine learning models that classified the data into diarrhea group or non-

150 diarrhea group. First, we split the data for 9 months (from April 2017 to December 2017) into the training dataset, and data for 3 months (from January 2018 to March 2018) into the test dataset. Next, based on the training dataset, we developed the random forest, SVM, and RBF network model, using R libraries named "randomForest" (version 4.6.14), “e1071” (version 1.7.2), and "RSNNS" (version 0.4.11), respectively.

By calculating the area under the curve (AUC) of the receiver operating characteristic curve using 5-fold cross-validation (DuBrava et al., 2017; Le et al., 2019b; Le, Ho \& Ou, 2017; Ukita, Yoshida \& Ohki, 2019), we performed feature selection and hyperparameters optimization to determine the models. Calculation of AUC was performed using an R library named 'ROCR' (version 1.0.7). The sets of hyperparameters were determined so that the AUC calculated by the cross-validation was optimized. Feature selection was performed using the backward elimination method for the variables filtered by statistical significance between the diarrhea group and nondiarrhea group within the training dataset (Tangaro et al., 2015; Milošević et al., 2017). According to the final AUC of each model, we selected the optimal model for the following analysis. The selected model was also validated using the independent test dataset.

\section{Visualization of variables' nature}


166 We visualized the variable importance in the selected model. We also visualized the association

167

168

169

170

171

172

173

174

175

176

177

178

179

180

181

182

183

184

185

186

187

188

189

190

191

192

193

194

195

196

197

198

199

200

201

202

203

204

205

between important variables and the outcome by partial dependence plots using an R library named "pdp" (version 0.7.0).

\section{Results}

\section{Participant characteristics}

Three hundred twenty-three patients with acute cerebral infarction were potentially eligible for inclusion in the present study, and after applying the exclusion criteria, 218 patients were included (Figure 1).

Table 1 shows the descriptive data for the diarrhea and non-diarrhea groups. Among the 218 patients, 48 had diarrhea during the observation period. In the diarrhea group, the test of CDI was performed in $12(25 \%)$ patients. Among them, $2(4 \%)$ patients were positive for the GDH antigen and none were positive for the $\mathrm{CD}$ toxin. The time of onset of nosocomial diarrhea ranged from 4 to 45 days from admission. There were no missing data for each variable. The patients in the diarrhea group were older, had a higher mRS score, had higher rates of antibiotic, tube feeding, and H2RA use. They also had longer duration of antibiotic and tube feeding use than those in the non-diarrhea group.

The VIF for each variable was $<2$, indicating there was no multicollinearity.

\section{Model selection}

Comparison of the model performance is shown in Table 2. Three variables (sex, H2RA use days, and PPI use days) were not significant between the diarrhea group and non-diarrhea group within the training dataset, which is similar to the results of the whole dataset shown in Table 1. Because eliminating some variables improved the performance, these variables were not used in the following analysis. The optimal hyperparameters were also determined for each model and used in the following analysis.

The 3 models achieved almost the same AUC on the 5 -fold cross-validation. The random forest model achieved a higher performance than the other 2 models. In addition, visualization of variable importance and partial dependence plots are widely used in the random forest model (Bair et al., 2013; DuBrava et al., 2017; Kimura et al., 2019; Halladay, Sillner \& Rudolph, 2018), unlike SVM or RBF network. Therefore, among the 3 models, we selected the random forest model for the following analysis.

The random forest model also achieved an AUC of 0.83 in the dependent test dataset, which was suitable for the following discussion regarding each variable.

\section{Variable importance}

Variable importance according to the mean decrease of Gini coefficient is shown in Figure 2.

The order of importance was as follows: tube feeding use days, mRS score, antibiotic use days, age, and sex. 


\section{Partial dependence plots}

207 Partial dependence plots of each variable are shown in Figure 3. The value of the y-axis in the

208 Figure was calculated using the partial dependence function, which approximately represented

209 the probability of nosocomial diarrhea.

210 Tube feeding use had an almost inverse U-shaped association with the outcome; the use of a

211 feeding tube drastically increased the risk of nosocomial diarrhea in the first few days, but

212 gradually decreased it thereafter. Overall, patients without use of tube feeding had a lower risk

213 than those with such use. The mRS score increased the risk of nosocomial diarrhea with a

214 convex downward and increasing association; especially patients with an mRS score $>3$ had a

215 high risk of nosocomial diarrhea. Antibiotic use had convex upward association; the use of

216 antibiotics rapidly increased the risk in the first few days, and slowly increased it thereafter.

217 Patients without use of antibiotics had a lower risk than those with such use. The association

218 between age and nosocomial diarrhea was convex downward and increasing; especially patients

219 aged $>90$ years had a high risk of nosocomial diarrhea. Male sex slightly increased the risk of

220 nosocomial diarrhea.

221

\section{Discussion}

223 In our study, the random forest model achieved high performance. The model showed that tube 224 feeding use days, mRS score, antibiotic use days, age, and sex were important, in this order.

225 Tube feeding use had an inverse U-shaped association with the outcome. The mRS score and age 226 showed a convex downward and increasing association. Antibiotic use showed a convex upward 227 association with the outcome. Male patients had a slightly higher risk of nosocomial diarrhea.

228 Tube feeding had the strongest association with the onset of nosocomial diarrhea in the analysis. 229 This result is consistent with that in previous studies (McFarland, 1995; Thorson, Bliss \& Savik, 230 2008; Arevalo-Manso et al., 2014). However, the model showed that when it was used for $>4$ 231 days, the risk conversely decreased, which could be a new finding. These results imply that if 232 nosocomial diarrhea occurred with prolonged use of tube feeding, clinicians should consider a 233 differential diagnosis other than diarrhea owing to tube feeding.

234 The mRS score, which was used for disease severity, was the second strongest factor associated 235 with nosocomial diarrhea. Patients with a low mRS score were often transferred on foot or with 236 light assistance, whereas those with a high mRS score were often transferred by a wheelchair or 237 on a stretcher. This might imply that activities of daily living, such as mode of movement, were 238 associated with the onset of nosocomial diarrhea. Previous research revealed that regular 239 exercise prevents episodes of diarrhea (Ma et al., 2014), and another study revealed that exercise 240 alters the composition and functional capacity of gut microbiota (Mailing et al., 2019). In 241 addition, the severity of disease was reported to have an association with nosocomial diarrhea in 242 previous studies (Kyne et al., 2002b; Thorson, Bliss \& Savik, 2008). These results may provide a 243 mechanism for the present findings.

244 Antibiotic use had an almost convex upward association with the outcome. The use of antibiotics 245 rapidly increased the risk of nosocomial diarrhea in the first 4 days. After 5 days of use, its use 
246 slowly increased the risk. Although previous studies revealed that antibiotic use is an important 247 risk factor for nosocomial diarrhea (McFarland, 1995; Arevalo-Manso et al., 2014; Eze et al., 248 2017), this convex upward association could be a new finding. A recent study showed that the 249 diversity of gut microbiome is affected by antibiotic administration and that the component of

250

251

252

253

254

255

256

257

258

259

260

261

262

263

264

265

266

267

268

269

270

271

272

273

274

275

276

277

278

279

280

281

282

283

284

285 gut microbiome changes with time (Bulow et al., 2018). The former rapid slope and latter gradual slope in the present study might arise owing to the effect of different microorganisms. Another recent study showed that the median of onset of CDI was about one week after antibiotic therapy (Thabit, Varugehese \& Levine, 2019), which does not conflict with our result, although our study had a limitation regarding CDI. Because our study might include a small number of patients with CDI, as discussed below, these results might apply mainly to antibioticassociated nosocomial diarrhea other than CDI. After all, the result of the present study might imply that clinicians should consider discontinuing antibiotics as soon as possible to prevent nosocomial diarrhea.

The association between age and nosocomial diarrhea was convex downward and increasing. Especially, age $>90$ years rapidly increased the risk of nosocomial diarrhea. This result is consistent with that of a previous study (McFarland, 1995).

Male patients had a slightly higher risk of nosocomial diarrhea than female patients. However, to our knowledge, there is no rational explanation of this result; it might imply that the collected data had some bias, and that the sex variable acted like a confounding factor. Number of days of PPI and H2RA use were not significantly different between the diarrhea group and the non-diarrhea group. They were also removed from the analysis by backward elimination, which implied that these variables were not important for prediction. Although previous studies showed that PPI and H2RA use were independent risk factors for nosocomial diarrhea (Eze et al., 2017), their importance was relatively low in the present study. The insignificance of antacid drugs was also observed in another study focusing on CDI (Thabit, Varugehese \& Levine, 2019). These results might imply that the effect of acid suppression therapy on risk of nosocomial diarrhea is lower than those of other risk factors, such as tube feeding, disease severity and antibiotics.

The primary limitation is that the causes of diarrhea were not precisely diagnosed and not included in the analysis. In particular, the present study included only 2 GDH antigen positive patients and no patients who were positive for CD toxin. Even considering the low sensitivity of our diagnosis tool for CDI (Kawada et al., 2011; Kosai et al., 2017; Morinaga et al., 2018), this rate is lower than that reported in hospitals in the US, where there were $20-30 \%$ cases of nosocomial antibiotic-associated diarrhea (McDonald et al., 2018). We considered that there are two rational explanations for this result. The first is that there were many patients with CDI overlooked in the study. A systematic review shows that prevalence of CDI in Japanese hospitals is lower than that of US and European countries because of under-diagnosis (Riley \& Kimura, 2018). Another study shows that numerous patients with CDI are being overlooked due to inadequate diagnostic testing in Japan (Kato, 2019). These studies might support the first explanation. Another explanation is that there was actually a small number of patients with CDI. 
286

287

288

289

290

291

292

293

294

295

296

297

298

299

300

301

302

303

304

305

306

307

308

309

310

311

312

313

314

315

316

317

318

319

320

321

322

323

324

As reported in a previous study (Morii et al., 2018), antimicrobial stewardship was widely

implemented in Showa General Hospital from October 2010. Because antimicrobial stewardship greatly reduces the frequency of CDI (Baur et al., 2017), the number of patients with CDI might actually be low, as reported in Table 1 . However, these hypotheses could not be verified retrospectively, and we consider this point to be the primary limitation. Other limitations are as follows: the present study was conducted in a single center, so external validity was not confirmed; the types and amounts of medications and tube feeding were not considered; and some risk factors or confounding factors, such as serum albumin level, were not considered in the analysis.

\section{Conclusions}

We revealed the degree of importance and temporal pattern of the influence of several risk factors for nosocomial diarrhea, such as tube feeding, mRS score, antibiotic use, and age. These findings could help clinicians manage nosocomial diarrhea.

\section{Acknowledgements}

We thank Drs. Eri Fukao and Yutaka Honma for their helpful advice on the methods of neurological assessments. We also thank Dr. Jumpei Ukita for his helpful advice on the machine learning analysis.

\section{References}

Adams HP Jr, Bendixen BH, Kappelle LJ, Biller J, Love BB, Gordon DL, Marsh EE 3rd. 1993. Classification of subtype of acute ischemic stroke. Definitions for use in a multicenter clinical trial. TOAST. Trial of Org 10172 in Acute Stroke Treatment. Stroke 24:35-41. DOI:

https://doi.org/10.1161/01.str.24.1.35

Arevalo-Manso JJ, Martinez-Sanchez P, Juarez-Martin B, Fuentes B, Ruiz-Ares G, Sanz-Cuesta BE, Parrilla-Novo P, Diez-Tejedor E. 2014. Enteral tube feeding of patients with acute stroke: when does the risk of diarrhea increase? Internal Medicine Journal 44:1199-1204. DOI: https://doi.org/10.1111/imj.12586

Bair E, Ohrbach R, Fillingim RB, Greenspan JD, Dubner R, Diatchenko L, Helgeson E, Knott C, Maixner W, Slade GD. 2013. Multivariable modeling of phenotypic risk factors for first-onset TMD: the OPPERA prospective cohort study. The Journal of Pain 14:T102-T115. DOI: https://doi.org/10.1016/i.jpain.2013.09.003

Bauer TM, Lalvani A, Fehrenbach J, Steffen I, Aponte JJ, Segovia R, Vila J, Philippczik G, Steinbrückner B, Frei R, Bowler I, Kist M. 2001. Derivation and validation of guidelines for stool cultures for enteropathogenic bacteria other than Clostridium difficile in hospitalized adults. JAMA 285:313-319. DOI: https://doi.org/10.1001/jama.285.3.313

Baur D, Gladstone BP, Burkert F, Carrara E, Foschi F, Döbele S, Tacconelli E. 2017. Effect of antibiotic stewardship on the incidence of infection and colonisation with antibiotic-resistant 
325 bacteria and Clostridium difficile infection: a systematic review and meta-analysis. The Lancet

326 Infectious Diseases 17:990-1001. DOI: https://doi.org/10.1016/S1473-3099(17)30325-0

327 Breiman LL. 2001. Random forests. Machine Learning 45:5-32.

328 Bulow C, Langdon A, Hink T, Wallace M, Reske KA, Patel S, Sun X, Seiler S, Jones S, Kwon

329 JH, Burnham CA, Dantas G, Dubberke ER. 2018. Impact of amoxicillin-clavulanate followed by 330 autologous fecal microbiota transplantation on fecal microbiome structure and metabolic

331 potential. $m$ Sphere 3:e00588-18. DOI: https://doi.org/10.1128/mspheredirect.00588-18

332 Cho HH, Lee SH, Kim J, Park H. 2018. Classification of the glioma grading using radiomics

333 analysis. PeerJ 6:e5982. DOI: https://doi.org/10.7717/peerj.5982

334 Ding J, Yang A, Wang J, Sagan V, Yu D. 2018. Machine-learning-based quantitative estimation

335 of soil organic carbon content by VIS/NIR spectroscopy. PeerJ 6:e5714. DOI:

336 https://doi.org/10.7717/peerj.5714

337

338

DuBrava S, Mardekian J, Sadosky A, Bienen EJ, Parsons B, Hopps M, Markman J. 2017. Using

339 random forest models to identify correlates of a diabetic peripheral neuropathy diagnosis from electronic health record data. Pain Medicine 18:107-115. DOI:

340

341 https://doi.org/10.1093/pm/pnw096

342 Eze P, Balsells E, Kyaw MH, Nair H. 2017. Risk factors for Clostridium difficile infections - an overview of the evidence base and challenges in data synthesis. Journal of Global Health 7:010417. DOI: https://doi.org/10.7189/jogh.07.010417

344 Fisher A, Rudin C, Dominici F. 2018. Variable importance for black-box, proprietary, or misspecified prediction models, using model class reliance. Available at https://arxiv.org/abs/1801.01489

347 Friedman JH. 2001. Greedy function approximation: A gradient boosting machine. Annals of 348 Statistics 29:1189-1232. DOI: https://doi.org/10.1214/aos/1013203451

349 Goldenberg JZ, Yap C, Lytvyn L, Lo CK, Beardsley J, Mertz D, Johnston BC. 2017. Probiotics 350 351 for the prevention of Clostridium difficile-associated diarrhea in adults and children. Cochrane Database Systematic Review 12:CD006095. DOI:

352 https://doi.org/10.1002/14651858.CD006095.pub4

354 Halladay CW, Sillner AY \& Rudolph JL. 2018. Performance of electronic prediction rules for prevalent delirium at hospital admission. JAMA Network Open 1:e181405. DOI:

https://doi.org/10.1001/jamanetworkopen.2018.1405

356 Hempel S, Newberry SJ, Maher AR, Wang Z, Miles JN, Shanman R, Johnsen B, Shekelle PG. 2012. Probiotics for the prevention and treatment of antibiotic-associated diarrhea: a systematic review and meta-analysis. JAMA 307:1959-1969. DOI: https://doi.org/10.1001/jama.2012.3507 Kato H, Senoh M, Honda H, Fukuda T, Tagashira Y, Horiuchi H, Chiba H, Suzuki D, Hosokawa N, Kitazono H, Norisue Y, Kume H, Mori N, Morikawa H, Kashiwagura S, Higuchi A, Kato H, Nakamura M, Ishiguro S, Morita S, Ishikawa H, Watanabe T, Kojima K, Yokomaku I, Bando T, 
365

366

367

368

369

370

371

372

373

374

375

376

377

378

379

380

381

382

383

384

385

386

387

388

389

390

391

392

393

394

395

396

397

398

399

400

401

402

403

Clostridioides (Clostridium) difficile infection burden in Japan: a multicenter prospective study. Anaerobe pii: S1075-9964(19)30046-0. DOI: https://doi.org/10.1016/j.anaerobe.2019.03.007 Kawada M, Annaka M, Kato H, Shibasaki S, Hikosaka K, Mizuno H, Masuda Y, Inamatsu T. 2011. Evaluation of a simultaneous detection kit for the glutamate dehydrogenase antigen and toxin $\mathrm{A} / \mathrm{B}$ in feces for diagnosis of Clostridium difficile infection. Journal of Infection and Chemotherapy 17:807-811. DOI: https://doi.org/10.1007/s10156-011-0267-5

Kosai K, Iwanaga Y, Akamatsu N, Okada Y, Kaku N, Uno N, Morinaga Y, Hasegawa H, Miyazaki T, Izumikawa K, Mukae H, Yanagihara K. 2017. Performance evaluation of the Verigene Clostridium difficile nucleic acid test, an automated multiplex molecular testing system for detection of C. difficile toxin. Journal of Infection and Chemotherapy 23:674-677. DOI: https://doi.org/10.1016/j.jiac.2017.07.002

Kimura N, Aso Y, Yabuuchi K, Ishibashi M, Hori D, Sasaki Y, Nakamichi A, Uesugi S, Fujioka H, Iwao S, Jikumaru M, Katayama T, Sumi K, Eguchi A, Nonaka S, Kakumu M, Matsubara E. 2019. Modifiable lifestyle factors and cognitive function in older people: a cross-sectional observational study. Frontiers of Neurology 10:401. DOI:

https://doi.org/10.3389/fneur.2019.00401

Kolominsky-Rabas PL, Weber M, Gefeller O, Neundoerfer B, Heuschman PU. 2001.

Epidemiology of ischemic stroke subtypes according to TOAST criteria: incidence, recurrence, and long-term survival in ischemic stroke subtypes: a population-based study. Stroke 32:27352740. DOI: https://doi.org/10.1161/hs1201.100209

Kyne L, Hamel MB, Polavaram R, Kelly CP. 2002a. Health care costs and mortality associated with nosocomial diarrhea due to Clostridium difficile. Clinical Infectious Diseases 34:346-353. DOI: https://doi.org/10.1086/338260

Kyne L, Sougioultzis S, McFarland LV, Kelly CP. 2002b. Underlying disease severity as a major risk factor for nosocomial Clostridium difficile diarrhea. Infection Control \& Hospital Epidemiology 23:653-659. DOI: https://doi.org/10.1086/501989

Le NQK, Ho QT, Ou YY. 2018. Classifying the molecular functions of Rab GTPases in membrane trafficking using deep convolutional neural networks. Analytical Biochemistry 555:33-41. DOI: https://doi.org/10.1016/j.ab.2018.06.011

Le NQK, Yapp EKY, Ou YY, Yeh HY. 2019a. iMotor-CNN: Identifying molecular functions of cytoskeleton motor proteins using 2D convolutional neural network via Chou's 5-step rule.

Analytical Biochemistry 575:17-26. DOI: https://doi.org/10.1016/j.ab.2019.03.017

Le NQK, Yapp EKY, Ho QT, Nagasundaram N, Ou YY, Yeh HY. 2019b. iEnhancer-5Step: Identifying enhancers using hidden information of DNA sequences via Chou's 5-step rule and word embedding. Analytical Biochemistry 571:53-61. DOI:

https://doi.org/10.1016/j.ab.2019.02.017

Le NQK, Ho QT, Ou YY. 2017. Incorporating deep learning with convolutional neural networks and position specific scoring matrices for identifying electron transport proteins. Journal of Computational Chemistry 38:2000-2006. DOI: https://doi.org/10.1002/jcc.24842 
404 Lewis SJ, Heaton KW. 1997. Stool form scale as a useful guide to intestinal transit time.

405 Scandinavian Journal of Gastroenterology 32:920-924. DOI:

406 https://doi.org/10.3109/00365529709011203

407 Ma C, Wu S, Yang P, Li H, Tang S, Wang Q. 2014. Behavioural factors associated with diarrhea

408 among adults over 18 years of age in Beijing, China. BMC Public Health 14:451. DOI:

409 https://doi.org/10.1186/1471-2458-14-451

410 Mailing LJ, Allen JM, Buford TW, Fields CJ, Woods JA. 2019. Exercise and the gut

411 microbiome: a review of the evidence, potential mechanisms, and implications for human health.

412 Exercise and Sport Sciences Reviews 47:75-85. DOI:

413 https://doi.org/10.1249/jes.0000000000000183

414 McFarland LV. 1995. Epidemiology of infectious and iatrogenic nosocomial diarrhea in a cohort

415 of general medicine patients. American Journal of Infection Control 23:295-305. DOI:

416 https://doi.org/10.1016/0196-6553(95)90060-8

417 McDonald LC, Gerding DN, Johnson S, Bakken JS, Carroll KC, Coffin SE, Dubberke ER,

418 Garey KW, Gould CV, Kelly C, Loo V, Shaklee Sammons J, Sandora TJ, Wilcox MH. 2018.

419 Clinical practice guidelines for Clostridium difficile infection in adults and children: 2017 update

420 by the infectious diseases society of America (IDSA) and society for healthcare epidemiology of

421 America (SHEA). Clinical Infectious Diseases 66:987-994. DOI:

422 https://doi.org/10.1093/cid/ciy149

423 Milošević M, Van de Vel A, Cuppens K, Bonroy B, Ceulemans B, Lagae L, Vanrumste B, Van

424 Huffel S. 2017. Feature selection methods for accelerometry-based seizure detection in children.

425 Medical \& Biological Engineering \& Computing 55:151-165. DOI:

426 https://doi.org/10.1007/s11517-016-1506-9

427 Morii D, Ichinose N, Yokozawa T, Oda T. Impact of an infectious disease specialist on

428 antifungal use: an interrupted time-series analysis in a tertiary hospital in Tokyo. 2018. Journal

429 of Hospital Infection 99:133-138. DOI: https://doi.org/10.1016/j.jhin.2018.01.003

430 Morinaga Y, Akamatsu N, Matsuda J, Tateno H, Tomaru T, Tanaka A, Morita S, Nakamura M,

431 Kato H, Annaka M, Masuda Y, Itakura Y, Inamatsu T, Yanagihara K. 2018. Diagnostic utilities

432 of a fully automated molecular test for toxigenic Clostridium difficile. Journal of Infection and

433 Chemotherapy 24:88-91. DOI: https://doi.org/10.1016/j.jiac.2017.09.003

434 Riley TV, Kimura T. 2018. The epidemiology of Clostridium difficile infection in Japan: a

435 systematic review. Infectious Diseases and Therapy 7:39-70. DOI:

436 https://doi.org/10.1007/s40121-018-0186-1

437 Tangaro S, Amoroso N, Brescia M, Cavuoti S, Chincarini A, Errico R, Inglese P, Longo G,

438 Maglietta R, Tateo A, Riccio G, Bellotti R. 2015. Feature selection based on machine learning in

439 MRIs for hippocampal segmentation. Computational and Mathematics Methods in Medicine

440 2015:814104. DOI: https://doi.org/10.1155/2015/814104

441 Tamune H, Ukita J, Hamamoto Y, Tanaka H, Narushima K, Yamamoto N. 2019. Efficient

442 prediction of vitamin $\mathrm{B}$ deficiencies via machine-learning using routine blood test results in 
443 patients with intense psychiatric episode. Available at

444 https://www.medrxiv.org/content/10.1101/19004317v1

445 Thabit AK, Varugehese CA, Levine AR. 2019. Antibiotic use and duration in association with

446 Clostridioides difficile infection in a tertiary academic medical center: a retrospective case-

447 control study. Anaerobe 59:126-130. DOI: https://doi.org/10.1016/j.anaerobe.2019.06.016

448 Thorson MA, Bliss DZ, Savik K. 2008. Re-examination of risk factors for non-Clostridium

449 difficile-associated diarrhoea in hospitalized patients. Journal of Advanced Nursing 62:354-364.

450 DOI: https://doi.org/10.1111/j.1365-2648.2008.04607.x

451 Ukita J, Yoshida T, Ohki K. 2019. Characterisation of nonlinear receptive fields of visual

452 neurons by convolutional neural network. Scientific Reports 9:3791. DOI:

453 https://doi.org/10.1038/s41598-019-40535-4

454 van Swieten JC, Koudstaal PJ, Visser MC, Schouten HJ, van Gijn J. 1988. Interobserver

455 agreement for the assessment of handicap in stroke patients. Stroke 19:604-607. DOI:

456 https://doi.org/10.1161/01.str.19.5.604

457 World Health Organization. Diarrhoea. Available at http://www.who.int/topics/diarrhoea/en/

458 (accessed June 18, 2019)

459 Zarbakhsh P, Addeh A. 2018. Breast cancer tumor type recognition using graph feature selection

460 technique and radial basis function neural network with optimal structure. Journal of Cancer

461 Research and Therapy 14:625-633. DOI: https://doi.org/10.4103/0973-1482.183561 
Figure 1

Flow chart of the study cohort.

Flow chart shows the number of included and excluded patients and the reasons for exclusion.

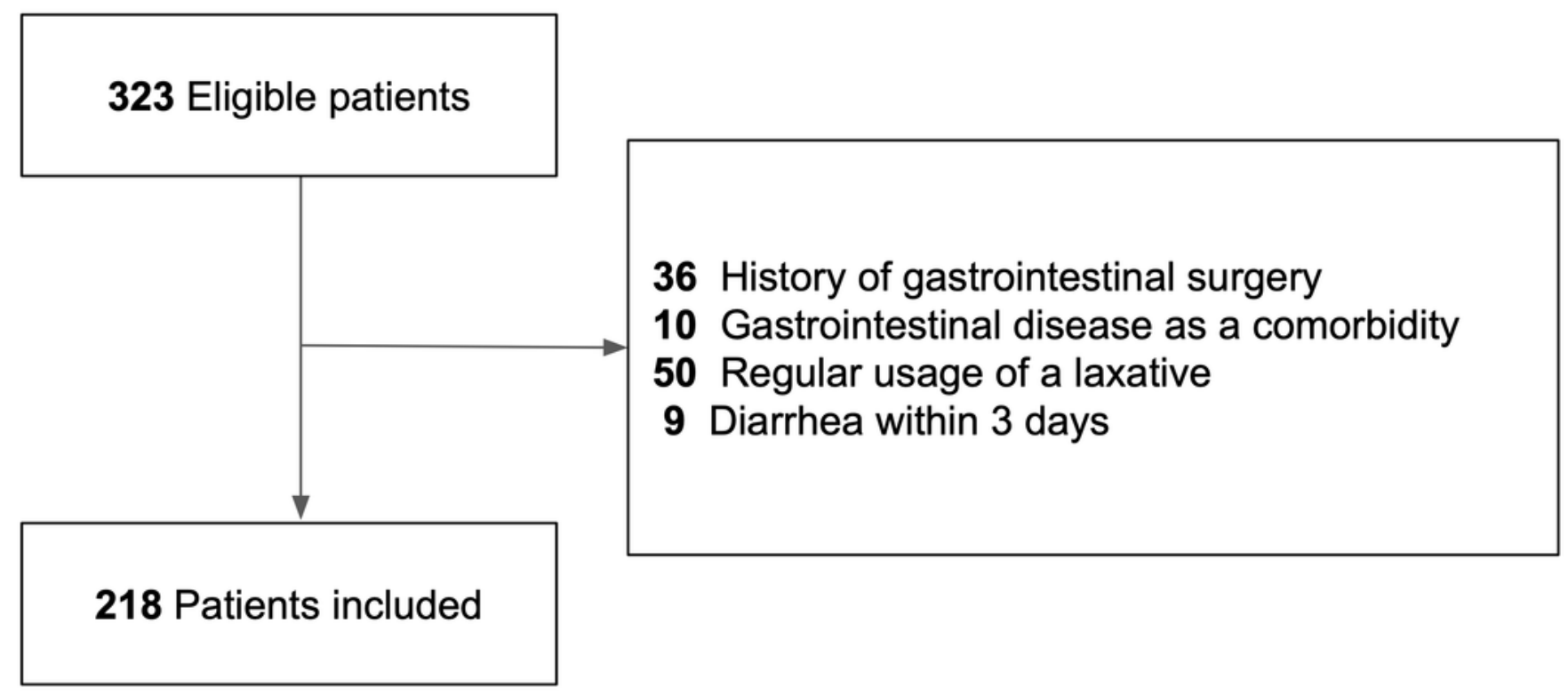


Figure 2

Variable importance according to mean decrease in Gini coefficient.

Bar graphs show the mean decrease in the Gini coefficient of each variable, which is considered as the index of importance. mRS = modified Rankin Scale. 


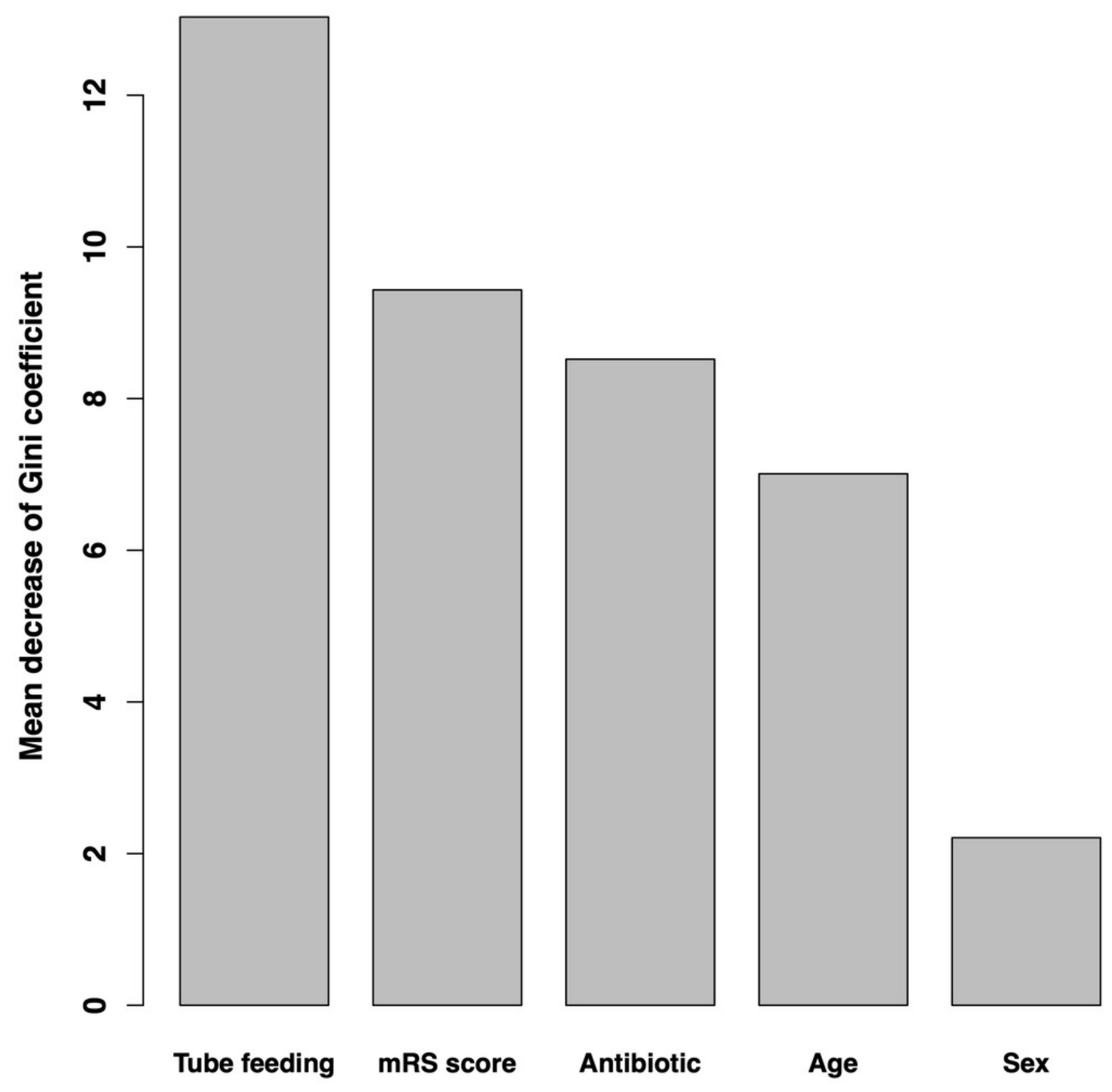


Figure 3

Partial dependence plots.

A)Temporal changes in the influence of tube feeding use. (B)The association between mRS score and influence. (C)Temporal changes in the influence of antibiotics use. (D)The association between age and influence. (E)The association between sex and influence. mRS $=$ modified Rankin Scale.

A

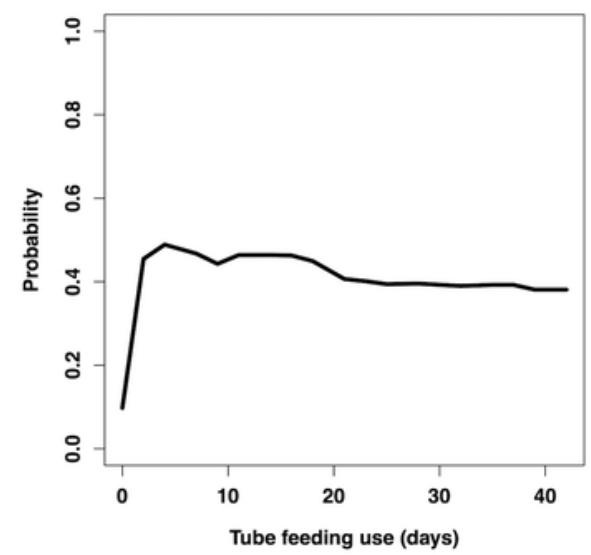

$\mathrm{D}$

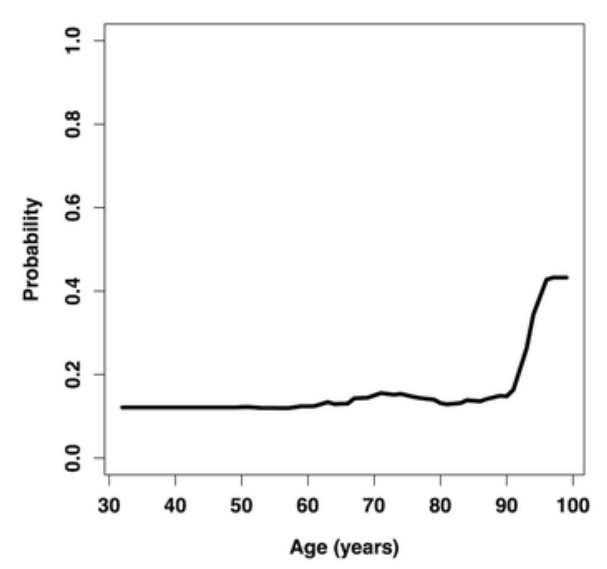

B

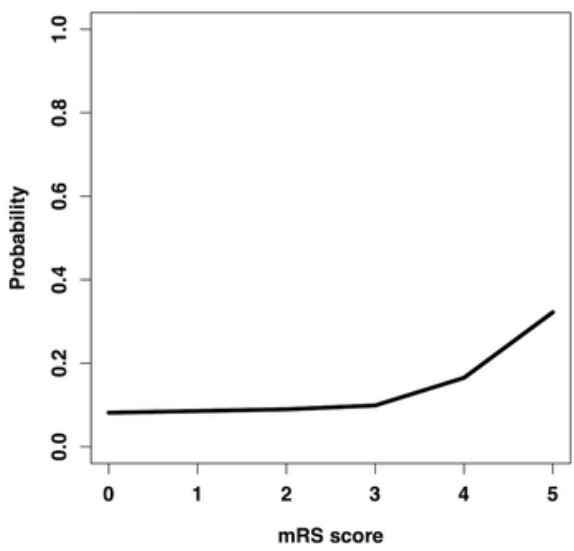

E

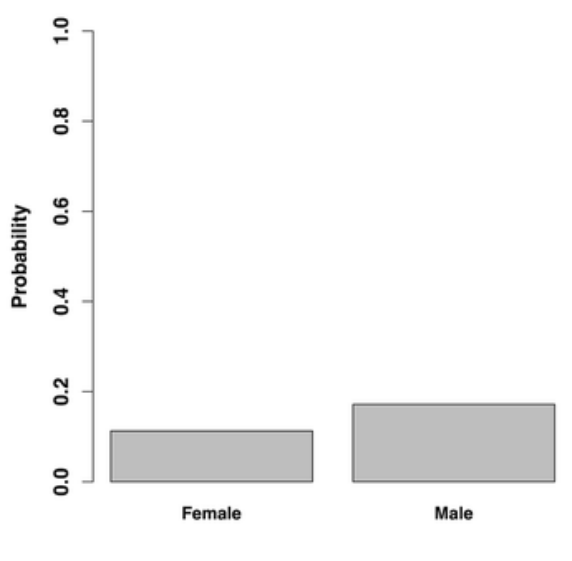

$\mathrm{C}$

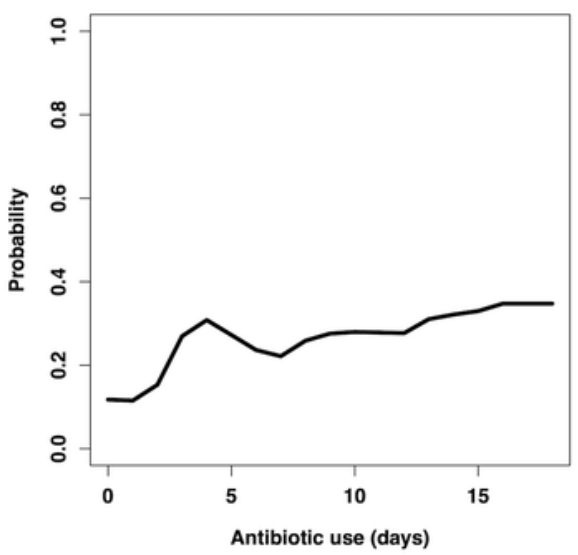




\section{Table 1 (on next page)}

Table 1 Characteristics of the study population.

Table 1 shows the characteristics of the patients. 
1 Table 1: Characteristics of the study population.

\begin{tabular}{|c|c|c|c|}
\hline Variable & $\begin{array}{l}\text { Diarrhea } \\
\text { group } \\
(n=48)\end{array}$ & $\begin{array}{l}\text { Non-diarrhea } \\
\text { group } \\
(\mathbf{n}=\mathbf{1 7 0})\end{array}$ & $P$ value \\
\hline Observational period, median (range), days & $13(4-45)$ & $17.5(5-59)$ & \\
\hline Age, mean (SD), years & $80.6(10.0)$ & $75.6(10.9)$ & $.004^{\mathrm{a}}$ \\
\hline mRS score, mean (SD) & $4.25(0.86)$ & $2.45(1.50)$ & $<.001^{\mathrm{a}}$ \\
\hline Male sex, n (\%) & $35(73)$ & $97(57)$ & $.047^{\mathrm{b}}$ \\
\hline Antibiotic use, n (\%) & $25(52)$ & $19(11)$ & $<.001^{\mathrm{b}}$ \\
\hline Number of days of use, median (range) & $3(0-18)$ & $0(0-14)$ & $<.001^{\mathrm{a}}$ \\
\hline Tube feeding use, $\mathrm{n}(\%)$ & $25(52)$ & $6(4)$ & $<.001^{b}$ \\
\hline Number of days of use, median (range) & $1(0-33)$ & $0(0-42)$ & $<.001^{\mathrm{a}}$ \\
\hline PPI use, n (\%) & $33(69)$ & $120(71)$ & $.81^{\mathrm{b}}$ \\
\hline Number of days of use, median (range) & $6(0-45)$ & $11(0-59)$ & $.09^{\mathrm{a}}$ \\
\hline H2RA use, n (\%) & $31(65)$ & $77(45)$ & $.02^{\mathrm{b}}$ \\
\hline Number of days of use, median (range) & $2(0-32)$ & $0(0-32)$ & $.36^{\mathrm{a}}$ \\
\hline \multicolumn{4}{|l|}{ Tests for Clostridioides difficile (CD) infection } \\
\hline Examination conducted, $\mathrm{n}(\%)$ & $12(25)$ & & \\
\hline $\mathrm{CD}$ toxin $\mathrm{A}$ and $\mathrm{B}$ positive, $\mathrm{n}(\%)$ & $0(0)$ & Not applicable & \\
\hline GDH antigen positive, $\mathrm{n}(\%)$ & $2(4)$ & & \\
\hline No examination conducted, $\mathrm{n}(\%)$ & $36(75)$ & & \\
\hline
\end{tabular}

2 H2RA = histamine 2-receptor antagonist; $\mathrm{mRS}=$ modified Rankin Scale; PPI = proton pump

3 inhibitor. GDH = glutamate dehydrogenase.

$4 \quad$ a Student's $t$ test. ${ }^{\text {b }}$ Chi-squared test. 
Table 2 (on next page)

Performance of the models.

Table 2 shows the performance of the machine learning models. 
1 Table 2: Performance of the models.

\begin{tabular}{ccll}
\hline & AUC & Variables eliminated & Hyperparameters \\
\hline $\begin{array}{l}\text { 5-fold cross-validation } \\
\text { Random forest }\end{array}$ & 0.944 & PPI, H2RA & $\begin{array}{l}\text { Number of features: } 1 \\
\text { Number of trees: } 500\end{array}$ \\
SVM & 0.937 & H2RA & $\begin{array}{l}\text { Gamma: } 0.0063 \\
\text { Cost: } 0.016\end{array}$ \\
$\begin{array}{l}\text { RBF network } \\
\text { Independent test dataset } \\
\text { Random forest }\end{array}$ & 0.934 & Sex & Size of hidden layer: 22 \\
\hline
\end{tabular}

$2 \mathrm{SVM}=$ Support vector machine; $\mathrm{RBF}=$ Radial basis function; $\mathrm{AUC}=$ Area under the curve 